\title{
Féeries
}

Études sur le conte merveilleuX, XVII $-\mathrm{XIX}{ }^{\mathrm{e}}$ siècle

$11 \mid 2014$

L'illustration des contes

\section{Les illustrations des Contes et satires de Voltaire par Moreau le Jeune, pour la première édition des Euvres complètes de Voltaire (1784-1789)}

Illustrating the Tales and Satires of Voltaire: Moreau le Jeune, for the First Edition of the Complete Works of Voltaire (1784-1789)

\section{Linda Gil}

\section{OpenEdition}

Journals

Édition électronique

URL : http://journals.openedition.org/feeries/948

DOI : 10.4000/feeries.948

ISSN : 1957-7753

Éditeur

UGA Éditions/Université Grenoble Alpes

Édition imprimée

Date de publication : 19 décembre 2014

Pagination : 221-243

ISBN : $978-2-84310-281-3$

ISSN : $1766-2842$

\section{Référence électronique}

Linda Gil, «Les illustrations des Contes et satires de Voltaire par Moreau le Jeune, pour la première édition des (Euvres complètes de Voltaire (1784-1789) », Féeries [En ligne], 11 | 2014, mis en ligne le 19 décembre 2015, consulté le 29 octobre 2020. URL : http://journals.openedition.org/feeries/948 ; DOI : https://doi.org/10.4000/feeries.948 


\section{Linda Gil}

Université Paris-Sorbonne et Università degli Studi di Roma Tre

\section{LES ILLUSTRATIONS DES CONTES ET SATIRES DE VOLTAIRE PAR MOREAU LE JEUNE, POUR LA PREMIÈRE ÉDITION DES CEUVRES COMPLÈTES} DE VOLTAIRE (1784-1789)

$E$ $N$ I764, naissait la tradition éditoriale du regroupement des "romans et contes ${ }^{\mathrm{I}}$ ", contemporaine de la première édition d'un recueil élaboré par Voltaire lui-même, les Contes de Guillaume Vadé. Avant cette date, les premiers récits voltairiens étaient regroupés dans des volumes de Mélanges, catégorie générique hybride qui pouvait accueillir des textes narratifs, mais aussi des poèmes, des pamphlets ou des essais. Voltaire sembla agréer l'idée d'un recueil composé a posteriori, suggérant à un libraire en 1767 : «Un petit recueil de contes serait peutêtre plus aisé à faire. Zadig, Candide, Memnon, Le Bramin et la vieille, la Fée Urgèle, etc. pourraient faire deux volumes que vous vendriez bien si je ne me trompe ${ }^{2}$.» Il est même favorable à l'idée d'orner l'édition d'illustrations : "Il me semble que vous pourriez faire une jolie édition avec des estampes, de tous les contes en vers et en prose ${ }^{3}$.» Dans les années I770, les éditions se succèdent, dont certaines comportent des figures ${ }^{4}$. La première

I. Le libraire parisien Panckoucke en avait suggéré l'idée à Voltaire. La première édition semble bien avoir été réalisée à Paris, peut-être chez un autre libraire cependant.

2. Lettre de Voltaire à Lacombe, 27 avril I767, D I4I46, Correspondence and related documents, T. Besterman (ed.), 2nd ed., Genève, Banbury \& Oxford, Institut et Musée Voltaire \& Voltaire Foundation, 1968-1977, D I6267. Pour la suite des références à la correspondance de Voltaire, nous donnons désormais simplement le numéro de la lettre, précédé de la lettre $\mathrm{D}$, selon la convention établie par l'éditeur.

3. Voltaire à Lacombe, I4 septembre I767, D I4423.

4. Nous utilisons la classification issue du projet bibliographique cr8.net, réalisé par le Centre International d'Études du XvıII ${ }^{\mathrm{e}}$ siècle de Ferney-Voltaire, consultable en ligne : <http://cı8.net/ vo/vo_pages.php?nom=vo_oe_I8_liste>; R72 Romans, ou contes philosophiques; qui forment un corps d'ouvrages, Londres [Lausanne, Grasset], I772, 2 tomes, $8^{\circ}$; R73 Romans et contes philosophiques, Londres, I773, 2 tomes, $\mathrm{I}^{\circ}$; R75 Romans et contes philosophiques, Londres [Rouen, Machuel?], I775, 2 tomes, $\mathrm{I}_{2}^{\circ} ; \mathrm{R} 77$ Romans et contes philosophiques, Londres, $\mathrm{I} 777,2$ tomes, $\mathrm{I}^{\circ}{ }^{\circ}$ ou $8^{\circ} ; \mathrm{R} 78 \mathrm{~B}$ Romans 
édition posthume des Euvres complètes de Voltaire, imprimée à Kehl, en Allemagne, entre I78I et I789 sous la double direction de Beaumarchais et de Condorcet, instaure la séparation du corpus narratif en vers et en prose. Elle présente un volume de Contes en vers et deux volumes de Romans 5 . L'élaboration du premier recueil passe par une phase d'incertitude générique, puisque le plan d'édition prévoyait une catégorie pour la "poësie didactique, ou narration, ou érotique; les contes charmans, le Vadé qui sont aussi en trop petit nombre. Les historiettes ou allégories ${ }^{6} »$. Le tome I4 rassemble finalement les contes et les satires en vers, ainsi que des "poésies mêlées", selon une logique poétique et un souci éditorial de «ne pas laisser tout cela éparpillé de volumes en volumes, comme dans les anciennes édition ${ }^{7} »$. Le recueil comporte quatre œuvres illustrées entre 1782 et 1787 par Jean-Michel Moreau, dit "le Jeune» : il s'agit de trois contes relevant de l'esthétique du merveilleux, Ce qui plaît aux dames, Gertrude ou l'Éducation d'une fille et La Bégueule, bien que le deuxième texte s'y rattache de façon problématique. Le quatrième texte est un conte moral en vers, classé dans les écrits satiriques ${ }^{8}$. Si certaines des gravures réalisées pour les «romans» sont régulièrement rééditées, ce n’est pas le cas de notre corpus. L'édition des Romans et contes en vers en prose, présentée par E. Guitton en 1994, reproduit certaines des illustrations les plus célèbres, celles des contes en prose, sans en retenir aucune pour les contes en vers. Plus généralement, l'illustration de l'œuvre de Voltaire a peu mobilisé jusqu’à présent l'attention des spécialistes. Les deux dictionnaires de référence sur Voltaire ne comportent aucune entrée relative à cette question'. Pourtant, l'illustration fait partie de l'histoire éditoriale et culturelle des textes voltairiens, tout comme l'histoire de la production, de la publication et de la réception des textes ${ }^{\text {10 }}$. Elle témoigne d'une histoire

et contes, Bouillon, Société typographique, 1778,3 tomes, $8^{\circ}$, qui comporte 57 figures hors texte de Monnet, Marillier, Moreau le Jeune, et I3 vignettes par Monnet; R78BF Romans et contes, Bâle, Flick, 1778,2 tomes.

5. Il s'agit des tomes 44 et 45 .

6. Plan d'une édition des œuvres de M. de Voltaire, IMV, Ms AS 6or. Voir A. Brown et A. Magnan, "Aux origines de l'édition de Kehl. Le Plan Decroix-Panckoucke de 1777 ", Cahiers Voltaire, nº 4 , 2005, p. 83-I24.

7. $\mathrm{I}^{\text {er }}$ juin $\mathrm{I782}$, Ruault à Decroix, collection particulière.

8. La satire est une catégorie générique problématique, comme le rappelle J. Goldzink dans la préface qu'il vient de donner à un recueil d'Écrits satiriques de Voltaire, Paris, Flammarion, 2014.

9. R. Trousson et J. Vercruysse (dir.), Dictionnaire général Voltaire, Paris, H. Champion, 2003 et J. Goulemot, A. Magnan et D. Masseau (dir.), Inventaire Voltaire, Paris, Gallimard, 1995.

Io. Voir C. Martin, Dangereux suppléments. L'illustration du roman en France au dix-huitième siècle, Louvain-Paris-Dudley (MA), Peeters, coll. «La République des Lettres», 2005 et A. M. Bassy, 
de la lecture, programmant et orientant leur réception. Par son travail de sélection, d'illustration et d'interprétation graphique, chaque illustrateur se distingue par sa sensibilité esthétique et littéraire. L'ensemble du travail d'illustration résulte d'une sélection dans le corpus des contes et récits voltairiens, porteuse de sens. L'illustration des contes réalisée par Moreau s'inscrit dans un geste de plus grande portée : le dessinateur a choisi d'illustrer l'ensemble de la fiction voltairienne. Outre les corpus dramatique et narratif, son attention s'est également portée sur les deux grands poèmes de Voltaire, l'un épique, La Henriade, l'autre burlesque, La Pucelle. Le corpus que nous avons choisi d'interroger pose une série de questions : comment s'est organisée la collaboration éditoriale avec la Société littéraire typographique fondée par Beaumarchais? Quels choix Moreau a-t-il opérés à partir du texte voltairien, compte-tenu des données de la nouvelle édition? Quelles lectures son travail de création esthétique propose-t-il de l'univers du conte, à travers le dialogue entre figures et texte littéraire?

\section{Une collaboration ouverte}

Seuls trois portraits de l'auteur, signés Largillière, Latour et Houdon, devaient initialement illustrer la grande édition, selon l'annonce du premier Prospectus publié en janvier $178 \mathrm{I}^{\mathrm{II}}$. L'édition, dont la livraison s'achève en 1789 , comporte finalement cent huit gravures, la plupart réalisées par Jean-Michel Moreau, dit «le Jeune», exécutées à ses frais, publiées en livraisons et dont il céda des épreuves à Beaumarchais ${ }^{12}$.

Né en mars I74I dans une famille de modestes artistes parisiens, Moreau étudia d'abord le dessin à l'atelier du dessinateur et graveur parisien Le Bas, avant de travailler avec le peintre Le Lorrain, qu'il accompagna à Saint-Pétersbourg ${ }^{13}$. En I770, de retour à Paris, il est nommé dessinateur

«Le texte et l'image», dans R. Chartier et H.-J. Martin (dir.), Histoire de l'édition française, tome 2, Le livre triomphant 1660-I830, Paris, Fayard, 1990, p. 173-200.

II. Société littéraire typographique, Prospectus pour l'édition des Euvres complètes de M. de Voltaire, BNF, Fr. 22188, f. I4I-I60, édité par J. Vercruysse dans «Le Prospectus de l'édition de Kehl. Écran mythificateur", dans Le Livre du monde et le Monde des livres. Mélanges en l'honneur de F. Moureau, Paris, Presses de l'université Paris-Sorbonne, 20I2, p. I083-1094.

I2. Outre ceux de Voltaire, que nous venons de citer, l'édition reproduit d'autres portraits qui ne sont pas de Moreau le Jeune.

I3. Sur l'œuvre gravé de Moreau le Jeune, on peut consulter les monographies qui lui ont été consacrées: E. Bocher, Les Gravures Françaises du XVIII Siècle ou Catalogue Raisonné des Estampes, Vignettes, Eaux-Fortes, de I700 à I800, Sixième fascicule, Jean-Michel Moreau Le Jeune, XII, Paris, 
des Menus-Plaisirs du Roi et devient membre de l'Académie royale de peinture et de sculpture. En I773, il entre dans l'univers du livre, entamant parallèlement une carrière d'illustrateur : "Un illustrateur nouveau et de premier ordre, qui devait voir pendant cinquante ans la seule annonce de ses "figures" assurer dans la librairie de toute l'Europe le débit et la fortune d'un ouvrage", rappellent les frères Goncourt dans L'Art du XVIII siècle $e^{14}$. Lorsqu'il décide de se consacrer à l'œuvre de Voltaire, Moreau est donc un artiste célèbre. Il a déjà illustré des œuvres littéraires classiques, de grands textes du patrimoine culturel, mais aussi des ouvrages modernes de premier plan, comme l'Encyclopédie ou La Nouvelle Héloïse. Moreau aborde Voltaire, si l'on peut dire, par la fin. En effet, il se signale tout d'abord par une gravure célèbre entre toutes : l'estampe commémorative du "Couronnement de Voltaire» sur la scène du Théâtre-Français le 30 mars I778, achevée en mars I782, devient rapidement une œuvre fondatrice de l'iconographie et du mythe voltairiens ${ }^{\text {15. }}$. Cette première gravure représente en quelque sorte la préface de l'œuvre consacrée à Voltaire par Moreau le Jeune, un corpus total d'environ deux cents planches.

Toutefois, dès 1775, Moreau avait entrepris un projet d'illustration de La Henriade. Ce premier travail signale l'admiration personnelle de l'artiste pour l'écrivain, esquissant une lecture militante de l'œuvre. Moreau présente en effet au Salon de 178I "plusieurs dessins in-quarto, sujets de La Henriade qui formeront la première livraison des estampes proposées par souscription, pour l'ornement des Éditions de Monsieur de Voltaire ${ }^{16}{ }^{\prime}$, et qui seront livrées en I782. Au total, un corpus représentant une série de cent huit estampes, "dédiée à $S$. A. Monseigneur le prince de Prusse, destinée à orner les œuvres de Voltaire, se vendant chez l'auteur rue du Coq-Saint-Honorér ${ }^{17}$ » et dont s'occupa l'artiste pendant près de dix ans.

Moreau s'est donc mis au travail avant même que les conditions de sa collaboration avec les éditeurs soient définies. Sa richesse lui permet de

Morgand et Fatout, I882; A. M. Hind, Fragonard, Moreau le Jeune et les graveurs français de la fin du XVIII 'eiècle, Paris, Hachette \& C C', I9I4; M. J. F. Mahérault, L'oeuvre de Moreau-le-Jeune, Paris, A. Labitte, I880; A. Moureau, Les Moreau, Paris, Librairie de l'Art, I892.

I4. E. et J. de Goncourt, "Moreau», dans L'art du XVIII siècle, Notes et notules, éd. présentée par J.-L. Cabanès, Tusson, Éditions Du Lérot, 2007, vol. II, p. II5.

I5. Nous renvoyons à ce sujet à notre contribution «Voltaire et Rousseau face à la postérité dans les gravures de Moreau-le-Jeune», Cahiers Voltaire, n I2, 2013.

I6. Sur l'illustration de La Henriade par Moreau le Jeune, on peut consulter P. Mironneau et al., Voltaire et Henri IV, Paris, éd. Réunion des musées nationaux, $200 \mathrm{I}$.

17. Prospectus du 29 octobre 1782, G. et M. von Proschwitz, Beaumarchais et le «Courier de l'Europe", Documents inédits ou peu connus, Studies on Voltaire and the Eighteenth Century, vol. 274, Oxford, Voltaire Foundation, 1990, p. 721. 
ne pas dépendre des éditeurs. Les Goncourt précisent qu'il «peut, avec ses ressources propres, aborder les opérations de la vente sans intermédiaire, exploiter lui-même son talent, et s'en faire les gros revenus d'une grande entreprise ${ }^{18} »$. Comme d'autres illustrateurs de premier plan, il soustraite le travail de gravure et se consacre à la création et la composition de ses estampes, trait caractéristique de l'évolution de la profession dans la seconde moitié du XVIII ${ }^{\mathrm{e}}$ siècle ${ }^{19}$. La position de Moreau au sein de l'équipe est donc celle d'un artiste indépendant qui s'engage dans une collaboration éditoriale avec une équipe doublement dirigée, sur le plan typographique et financier, par Beaumarchais et, sur le plan scientifique et littéraire, par Condorcet. Cette association entre un artiste officiel de la monarchie française, pensionné par le roi, et les éditeurs clandestins d'une œuvre censurée, bannie hors des frontières du royaume pour cause d'impiété, témoigne de la posture paradoxale de cette geste éditoriale et de ses acteurs. S'il bénéficie des revenus d'une charge royale, les choix artistiques de Moreau font de lui un artiste des Lumières, qui met son talent au service des philosophes. Son admiration personnelle pour Voltaire le rattache à la communauté fraternelle des premiers voltairiens, réunis autour du combat pour porter et transmettre l'héritage du patriarche.

L'élaboration de l'œuvre de Moreau se fait donc dans une relative indépendance vis-à-vis du reste de l'équipe éditoriale. L'impression des volumes de textes et le travail du peintre apparaissent comme deux "affaires» distinctes mais complémentaires. C'est à ce titre que Moreau s'adresse à Beaumarchais en juillet 1782 pour lui demander son soutien logistique et médiatique. Il a commencé à réaliser les gravures en octobre I78I. Comme d'autres artistes de renom, il a opté pour le système de la souscription, qui permet d'obtenir les fonds nécessaires au lancement de l'entreprise, tout en conservant son indépendance vis-à-vis des éditeurs. Ainsi, les deux entreprises débutent quasiment au même moment, puisque l'année I78I est celle de l'installation et de la mise en fonctionnement de l'imprimerie de Kehl. Un avis paraît effectivement dans le Courier de l'Europe daté du 29 octobre 1782, sous la forme d'un Prospectus:

Pour cent Estampes in-4to, \& in-8vo. gravées d'après les dessins \& sous la direction de M. Moreau, dessinateur \& graveur du cabinet du Roi \& de son académie de Peinture, pour décorer la nouvelle édition de Voltaire, imprimée avec les caractères de Baskerville, par les soins de M. de Beaumarchais ${ }^{20}$.

I8. E. et J. de Goncourt, ouvr. cité, p. I35.

19. C. Martin et A. M. Bassy, ouvr. cité.

20. Prospectus du 29 octobre 1782, G. et M. von Proschwitz, ouvr. cité. 
Le Prospectus donne également des indications sur l'ordre de la création graphique, qui répond à la classification générique de l'édition, conformément au «Plan » élaboré par Decroix et agréé par Voltaire en $1777^{21}$ : d'abord le grand poème épique "dix estampes, pour être mises à la tête des chants de La Henriade. À la fin de l'année, on donnera la seconde livraison; les sujets seront tirés des pièces du Théâtre ${ }^{22} »$. En I783, le Courier de l'Europe fait paraître un nouvel avis, daté du ir juillet, qui confirme le caractère séparé des deux entreprises ${ }^{23}$. Moreau semble s'organiser progressivement, et apparaissent des formulaires imprimés et des avis de publications, dans la presse parisienne cette fois.

Les livraisons des estampes s'effectuent en plusieurs temps. On en dénombre au moins sept, une par an. Chaque nouvelle livraison est annoncée dans la presse, et les souscripteurs ou les acheteurs sont invités à venir retirer leurs estampes au domicile de Moreau, et à les faire relier à leurs frais. La renommée du dessinateur, ainsi que celle de l'édition prestigieuse des CEuvres complètes de Voltaire sont connues du public. La presse du temps commente l'événement. Par exemple, lors de la quatrième livraison, en septembre 1784, un article parait dans le Mercure de France:

Quatrième Livraison des Estampes pour les Euvres de Voltaire, in- $8^{\circ}$, À Paris, chez Moreau, Dessinateur et Graveur du Roi \& de son Cabinet, rue du Coq S. Honoré. La quatrième partie de cette riche Collection est dédiée à Son Altesse Royale le prince Frédéric Guillaume. Cette dédicace joint au mérite d'un juste hommage, celui d'un heureux à-propos. C'est un tribut que nos Arts rendent à l'un des plus grands Princes de l'Europe; \& il ne pouvait être offert par de plus dignes mains que par celles d'un de nos plus grands Artistes. [...] La fécondité de M. Moreau, dont l'œuvre est déjà très-nombreux, ne nuit point à la beauté de ses dessins; il produit beaucoup, \& il fait aussi bien que s'il produisait peu. Le plus grand éloge qu'on puisse donner à cette quatrième Livraison, c'est de dire qu'elle est digne des précédentes; \& quand cette Collection sera terminée, on dira que ce monument est digne du grand Homme à qui il est érigé24.

Y a-t-il eu collaboration littéraire ou esthétique entre l'artiste et les éditeurs? On l'ignore. Moreau semble avoir choisi lui-même ses sujets, avec une liberté rare dans le domaine de l'illustration du livre. Il a réalisé de façon autonome un travail de relecture, de sélection et de composition

21. Decroix, Plan d'une Édition des auvres de M. de Voltaire, ouvr. cité.

22. Prospectus du 29 octobre 1782 , G. et M. von Proschwitz, ouvr. cité.

23. Avis sur les éditions des CEuvres de Voltaire du II juillet I783, G. et M. von Proschwitz, ouvr. cité, p. 748-750.

24. Mercure de France daté du in septembre I784, nº 37, p. 94. 
à partir du corpus voltairien, avec lequel il engage un véritable dialogue interprétatif.

L'édition en format in $-8^{\circ}$ comporte soixante-dix volumes. Moreau a dessiné quarante-quatre figures pour le Théâtre, dix pour La Henriade, vingt-et-une pour La Pucelle d'Orléans, quatre pour les contes, quatorze pour les romans, soit un total de quatre-vingt-treize estampes ${ }^{25}$. Cette répartition appelle plusieurs remarques. Sur le plan générique, il a choisi d'illustrer uniquement les œuvres de fiction, dramatique et narrative, ainsi que les deux grands poèmes épiques et historiques. Les estampes s'adressent davantage à l'imagination du lecteur. Les images figuratives dominent, seules quelques-unes ont une fonction allégorique ou didactique.

La chronologie de la réalisation des gravures suggère également plusieurs pistes de réflexion. Le tableau que nous présentons ci-après permet d'en saisir le déroulement.

Les dates que nous donnons sont celles qui sont gravées au bas des vignettes. En l'absence d'autres éléments de datation, elles fournissent l'ordre de réalisation définitive des gravures, même si des états préparatoires, dont certains sont conservés au Cabinet des Estampes de la Bibliothèque nationale, ont pu être esquissés antérieurement. On remarque tout d'abord que Moreau n'a pas procédé systématiquement, en choisissant par exemple un ordre générique. La production s'étale sur huit ans, avec quinze illustrations datées de 1782 , douze en 1783 , huit en 1784 , dix-huit en 1785 , huit en $\mathrm{I} 786$, dix en 1787 , vingt-et-une en $\mathrm{I} 788$ et une dernière figure livrée en I789.

25. L. Gil, «Compléter l'œuvre de Voltaire : le supplément illustré de Moreau le Jeune pour l'édition de Kehl (I784-1789)", Journées Voltaire 20II, en ligne sur <http://voltaire.lire.ish-lyon.cnrs.fr/ spip.php?rubrique39> et "Jean-Michel Moreau, dit "le Jeune", illustrations de Candide», Cahiers Voltaire, $\mathrm{n}^{\circ}$ II, $20 \mathrm{I} 2$. 
LiNDA GIL

Tableau. - Les figures de Moreau le Jeune pour l'édition des Euvres complètes de Voltaire : chronologie.

\begin{tabular}{|c|c|c|c|c|c|}
\hline & Tragédies & Comédies & Poèmes & Contes en vers & Romans \\
\hline 1782 & $\begin{array}{c}\text { Mariamne } \\
\text { Zaïre } \\
\text { La Mort de César }\end{array}$ & & $\begin{array}{c}\text { La Henriade } \\
\text { (Io fig.) }\end{array}$ & Le Pauvre Diable & L'Ingénu \\
\hline 1783 & $\begin{array}{c}\text { Edipe } \\
\text { Brutus } \\
\text { Alzire } \\
\text { Mahomet } \\
\text { Mérope } \\
\text { Sémiramis } \\
\text { Oreste }\end{array}$ & $\begin{array}{c}\text { L'Indiscret } \\
\text { L'Enfant prodigue } \\
\text { Pandore }\end{array}$ & & $\begin{array}{l}\text { La Bégueule } \\
\text { Gertrude }\end{array}$ & \\
\hline I784 & $\begin{array}{c}\text { Rome sauvée ou } \\
\text { Catilina } \\
\text { Tancrède } \\
\text { Olimpie }\end{array}$ & $\begin{array}{c}\text { La Femme qui a raison } \\
\text { L'Écossaise }\end{array}$ & & & $\begin{array}{l}\text { Zadig (2 fig.) } \\
\text { Memnon }\end{array}$ \\
\hline 1785 & $\begin{array}{l}\text { Adélaïde } \\
\text { Amélie } \\
\text { Zulime } \\
\text { Les Scythes } \\
\text { Les Guèbres } \\
\text { Les Lois de Minos }\end{array}$ & \begin{tabular}{|c|} 
La Prude \\
Nanine \\
Le Droit du Seigneur \\
Charlot \\
Le Dépositaire \\
Socrate \\
Samson \\
La Princesse de Navarre \\
Le Temple de la Gloire \\
Tanis et Zélide \\
Le Baron d'Otrante \\
Les Deux Tonneaux \\
\end{tabular} & & & \\
\hline 1786 & $\begin{array}{c}\text { L'Orphelin de la Chine } \\
\text { Le Triumvirat } \\
\text { Sophonisbe } \\
\text { Dom Pedre } \\
\text { Les Pélopides } \\
\text { Irène }\end{array}$ & & & & $\begin{array}{c}\text { L'Ingénu } \\
\text { L'Homme aux } \\
\text { quarante écus }\end{array}$ \\
\hline I787 & Éryphile & & & $\begin{array}{c}\text { Ce qui plaît aux } \\
\text { dames }\end{array}$ & $\begin{array}{l}\text { Le Blanc et le Noir } \\
\text { Jeannot et Colin } \\
\text { Candide (4 fig.) } \\
\text { La Princesse de } \\
\text { Babylone (2 fig.) } \\
\end{array}$ \\
\hline I788 & & $\begin{array}{l}\text { La Pucelle } \\
\text { (2I fig.) }\end{array}$ & & & \\
\hline I789 & Agathocle & & & & \\
\hline
\end{tabular}




\section{L'illustration des Contes en vers et des Satires : choix littéraires et esthétiques}

Le corpus des Contes en vers est regroupé au tome quatorze de l'édition de Kehl. Le volume se compose de trois parties : les contes en vers proprement dits, au nombre de dix-sept, parmi lesquels les trois textes illustrés par Moreau, La Bégueule, Gertrude ou l'Éducation d'une fille et Ce qui plaît aux dames. Une deuxième section regroupe une catégorie intitulée "Satires", qui rassemble dix-sept textes narratifs, dont le quatrième texte illustré que nous avons retenu pour cette étude, Le Pauvre Diable. La dernière section est consacrée aux "Poésies mêlées». Au total donc, trente-trois pièces narratives figurent dans ce volume. Moreau a choisi d'en illustrer seulement quatre. Il y a là manifestement une volonté de sélection. Ce choix est-il littéraire ou esthétique? Il a réalisé une illustration pour chacun des quatre contes suivants : Le Pauvre Diable (I782), La Bégueule et Gertrude ou l'Éducation d'une fille (1783) et Ce qui plaît aux dames (1787). L’ordre éditorial des Contes en vers obéit à une logique chronologique, exactement inverse à l'histoire de la production des gravures. Les éditeurs ont partiellement respecté l'intégrité du recueil des Contes de Guillaume Vadé, qui comprenait, lors de sa publication en 1763 , vingttrois pièces. Ce qui plaît aux dames et Gertrude ou l'Éducation d'une fille en font partie. Les éditeurs en ont cependant détaché Le Pauvre Diable, qu'ils ont placé, comme nous l'avons signalé, dans une seconde section regroupant les «Satires».

La fiction du recueil des Contes de Guillaume Vadé, de son auteur et de son éditrice posthumes, redouble la fiction des contes. La Préface au recueil, donnée par Catherine, cousine de Guillaume, accentue la plaisanterie : retranscrivant une prétendue conversation avec son cousin à l'article de la mort, Catherine lui demande la permission de publier «les contes à dormir debout dont vous nous régalâtes l'année passée ${ }^{26} »$. L'auteur accepte à condition qu'on les regroupe sous le terme de "fadaises ${ }^{27}$ ». Voltaire n'a de cesse de jouer sur l'image d'un genre qu'il cultive tout en dénonçant sa fausseté. Le recueil s'ouvre avec Ce qui plaît aux dames, un conte merveilleux, un conte de fées. Voltaire, dès les premiers vers, fait l'éloge des contes, et se représente en patriarche à la veillée :

26. Voltaire, Préface aux Contes de Guillaume Vadé, Romans et Contes en vers et en prose, éd. présentée par É. Guitton, Paris, Flammarion, 1994, p. 339.

27. Ibid., p. 340 . 
Or maintenant que le beau dieu du jour

Des Africains va brûlant la contrée,

Qu'un cercle étroit chez nous borne son tour,

Et que l'hiver allonge la soirée;

Après souper, pour vous désennuyer,

Mes chers amis, écoutez une histoire

Touchant un pauvre et noble chevalier,

Dont l'aventure est digne de mémoire ${ }^{28}$.

Cependant, l'histoire du jeune chevalier, Messire Jean Robert, se situe dans un temps historique, celui du règne du roi Dagobert, le dernier des rois Mérovingiens, qui régna au VII ${ }^{\mathrm{e}}$ siècle. L'espace choisi n'est pas non plus indéterminé et lointain, comme dans les contes de fées traditionnels. L'aventure a pourtant lieu sur la route, dans un bois. Mais le lieu est précisément situé près de Lutèce, du côté de Charonne, sur le chemin de retour d'un voyage à Rome, où le jeune homme est allé se procurer des indulgences. La rencontre avec la jeune et belle Marthon a lieu «assez près de Lutèce/ Au coin d'un bois qui borde Charenton ${ }^{29}$ ». Cette esthétique rattache le conte au genre fantastique, tout en détournant les motifs traditionnels du conte merveilleux et des aventures galantes : la rencontre donne lieu à un marchandage trivial, et la belle accepte de vendre ses charmes pour vingt écus. Pendant leurs ébats, un moine de Saint-Denis vole le cheval et la bourse du voyageur, incapable désormais de payer la belle. La jeune paysanne porte l'affaire devant le roi, qui délègue le cas à sa femme, la reine Berthe. Condamné à mort pour viol, le jeune homme bénéficie, parce qu'il est beau garçon, d'une seconde chance. A-t-il autant d'esprit que de beauté? On lui propose une énigme. Il s'agit de trouver «ce que la femme en tous les temps désire ${ }^{30} »$. Robert a huit jours pour rapporter la réponse et obtenir sa grâce. Les sept premiers jours se passent à interroger toutes les femmes qu'il rencontre, en vain. Le huitième jour, le merveilleux intervient, sous la forme d'un mirage : croyant apercevoir un groupe de jeunes filles, le jeune homme s'approche pour «Les consulter sur la maudite affaire ${ }^{3 \mathrm{I}}$. Las! «En un moment tout disparaît, tout fuit. / Le jour baissait, à peine il était nuit; / Il ne vit plus qu'une vieille édentée ${ }^{32}$.» Celle-ci promet son aide, en échange d'une faveur qu'elle lui demandera en temps voulu. Elle révèle donc le secret de «ce qui plaît aux dames» à

28. Voltaire, Ce qui plaît aux dames, ibid., p. 367.

29. Ibid., p. 368.

3o. Ibid., p. 369.

3I. Ibid., p. 370.

32. Ibid. 
Robert qui s'en retourne à la cour de la reine Berthe. Au moment où il obtient sa grâce, surgit la vieille qui vient crier justice et réclamer son dû : elle veut épouser Robert et s'en faire aimer. Horrifié, le jeune homme crie au ridicule et à l'indignité.

C'est ce moment de crise que Moreau a choisi d'illustrer. Le dispositif éditorial place pourtant la gravure avant la lettre, c'est-à-dire en regard de l'incipit, comme c'est le cas pour tous les textes illustrés.

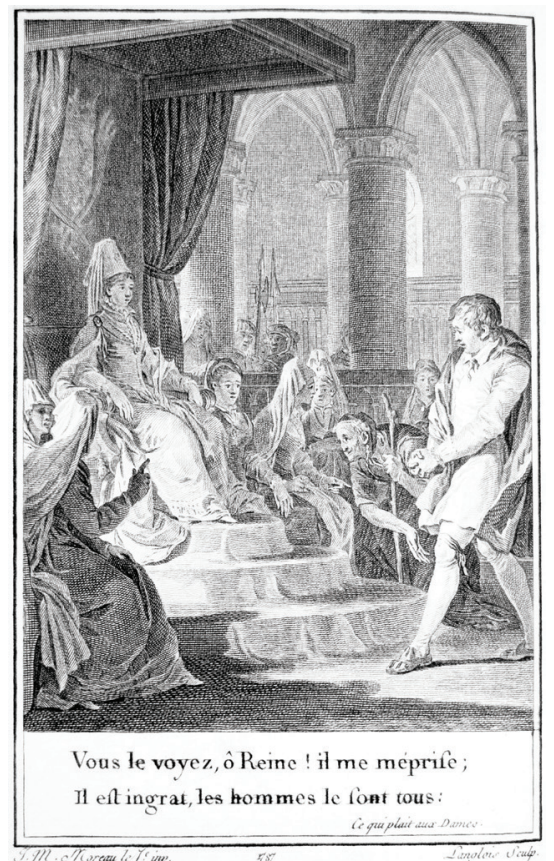

Fig. I. - «Vous le voyez, ô Reine! il me méprise; il est ingrat, les hommes le sont tous", Ce qui plâ̂t aux dames, 1787, CEuvres complètes de Voltaire.

Société littéraire typographique, I784, tome I4 (collection privée).

Dès lors, l'image se veut moteur de la réception de l'ensemble du texte, orientant l'horizon de lecture, invitant le lecteur au cœur de l'action. La gravure fait écho au titre, par la présence de la reine et de ses dames d'honneur, suggérant une intrigue galante. La scène gravée se situe dans un décor richement évoqué par le dessin, celui du château royal. L'architecture, comme souvent chez Moreau, est particulièrement travaillée ${ }^{33}$. La composition s'inspire d'un imaginaire médiéval, et la profondeur de l'espace est

33. À Saint-Pétersbourg, Moreau a travaillé à la création de décors de théâtre. 
donnée par la perspective qui suggère plusieurs plans. La scène d'audience royale est théâtralisée par la représentation du trône surélevé et coiffé d'un dais. Les rideaux, les marches drapées, l'écusson, les ogives gothiques, les colonnes, composent un décor particulièrement évocateur du luxe de la cour. Les deux personnages principaux, la reine et le jeune chevalier, se font face. La reine, entourée de ses dames d'atour, assises au pied du trône, vient de rendre la justice et d'accorder la grâce au chevalier. La fée Urgèle, métamorphosée en vieillarde, figure au second plan, courbée, appuyée sur un bâton. Le mouvement expressif de son bras et de son visage traduit l'acte oratoire. Ses paroles : "Vous le voyez, ô Reine! il me méprise; il est ingrat, les hommes le sont tous ${ }^{34} »$, servent de légende. Il s'agit d'un véritable coup de théâtre. Moreau a saisi cet instant de surprise par le jeu des regards qui convergent vers la vieille, par les mouvements de surprise des témoins, rendant la scène dynamique et célébrant, avec Voltaire, le pouvoir des fables.

Si les quatre contes illustrés par Moreau dans le recueil ont pour point commun d'être des contes en vers, ils appartiennent à des registres très différents. Le second récit, Gertrude ou l'Éducation d'une fille, n'est pas un conte de fées mais un conte moral, où le merveilleux prend la forme d'une initiation au plaisir, d'une éducation sentimentale et d'une apologie de l'amour. Ce conte fait également partie du recueil des Contes de Guillaume Vadé, et date donc de la même époque. La cohérence du recueil est assurée par l'incipit de ce récit, qui fait écho à l'épilogue du premier conte. Ce qui plaît aux dames s'achevait en effet par un éloge du conte merveilleux :

Ô l'heureux temps que celui des fables,

Des bons démons, des esprits familiers,

Des farfadets, aux mortels secourables!

On écoutait tous ces faits admirables

Dans son château, près d'un large foyer.

Le père et l'oncle, et la mère et la fille,

Et les voisins, et toute la famille,

Ouvraient l'oreille à monsieur l'aumônier,

Qui leur faisait des contes de sorcier.

On a banni les démons et les fées;

Sous la raison les grâces étouffées

Livrent nos cœurs à l'insipidité;

Le raisonner tristement s'accrédite;

34. Voltaire, Ce qui plaît aux dames, ouvr. cité, p. 373. 
On court, hélas! après la vérité :

Ah! croyez-moi, l'erreur a son mérite ${ }^{35}$.

Gertrude s'ouvre sur une reprise de l'image et de l'éloge, sur le mode d'une énonciation orale qui permet d'enclencher le récit :

Mes amis, l'hiver dure, et ma plus douce étude

Est de vous raconter les faits des temps passés.

Parlons ce soir un peu de madame Gertrude.

Je n'ai jamais connu de plus aimable prude :

Par trente-six printemps sur sa tête amassés,

Ses modestes appas n'étaient point effacés ${ }^{36}$.

Le récit met en scène la relation amoureuse que Dame Gertrude entretient secrètement avec un certain André. Alors que la mère affiche sa dévotion et sa chasteté, sa fille Isabelle découvre qu'elle a un amant, qu'elle reçoit la nuit. C'est la scène qu'a choisi d'illustrer Moreau (fig. 2).La jeune fille écoute à la porte de la chambre de sa mère et entend ces mystérieuses paroles, qui servent de légende à la gravure : «André, mon cher André, vous faites mon bonheur ${ }^{37}$.» Cette fois, la scène est en extérieur. On y voit la jeune fille, qui écoute à la porte d'un pavillon où sa mère aimait à se retirer, "un oratoire,/ Un boudoir de dévote où, pour se recueillir,/Elle allait saintement occuper son loisir ${ }^{38} »$. Le véritable sujet de la gravure est donc invisible. La représentation de la jeune fille entrouvre l'espace imaginaire de l'érotisme. Le décor est partagé par des lignes géométriques, architecture et végétation se complètent pour suggérer, peut-être, la rigidité des lignes géométriques et de la morale de convention d'un côté, l'harmonie des lignes arrondies de la végétation épanouie du jardin de l'autre. Moreau a suivi scrupuleusement les indications du texte pour la spatialisation de la scène. La figure de l'héroïne est animée d'un mouvement délicat. Les gestes de ses mains traduisent l'émotion qui la soulève à l'écoute des soupirs de sa mère, qu'elle prend pour l'expression d'une souffrance au premier instant, avant de se laisser gagner à son tour par le plaisir. Là encore, le texte a guidé le trait de Moreau :

Cependant elle hésite, elle approche en tremblant,

Posant sur l'escalier une jambe en avant,

Étendant une main, portant l'autre en arrière,

35. Ibid., p. 377-378.

36. Voltaire, L'Éducation d'une fille, ouvr. cité, p. 385.

37. Ibid., p. 387.

38. Ibid., p. 386. 
Le cou tendu, l'œil fixe, et le cœur palpitant, D'une oreille attentive avec peine écoutant ${ }^{39}$.

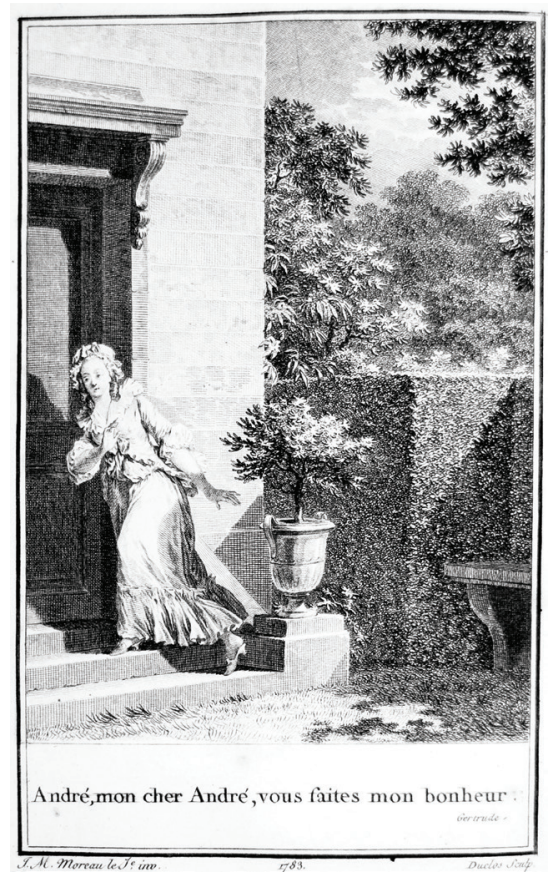

Fig. 2. - "André, mon cher André, vous faites mon bonheur», Gertrude ou l'Éducation d'une fille, I783, Euvres complètes de Voltaire.

Société littéraire typographique, I784, tome I4 (collection privée).

Isabelle, bonne élève, prend elle aussi un amant et découvre à son tour les joies de l'amour et du plaisir. La morale de l'histoire célèbre les vertus innocentes des plaisirs de l'amour, dénonce l'hypocrisie de la morale chrétienne qui condamne les relations amoureuses librement vécues et remet en question les notions de bien et de mal.

Avec La Bégueule, nous revenons au conte de fées, même si Voltaire l'a sous-titré «conte moral». Datant de $\mathrm{I} 772$, ce récit ne fait pas partie d'un recueil et se présente comme un apologue :

Dans ses écrits un sage italien

Dit que le mieux est l'ennemi du bien;

Non qu'on ne puisse augmenter en prudence,

En bonté d'âme, en talents, en science.

39. Ibid. 
Cherchons le mieux sur ces chapitres-là :

Partout ailleurs évitons la chimère.

Dans son état, heureux qui peut se plaire,

Vivre à sa place, et garder ce qu'il a!

La belle Arsène en est la preuve claire ${ }^{40}$.

Le récit met en scène une femme insatisfaite de son mariage, comme le sera Madame Bovary. Elle demande à sa bonne fée Aline d'intervenir. Celle-ci la transporte au royaume des dieux, inspiré d'un imaginaire de l'Antiquité.

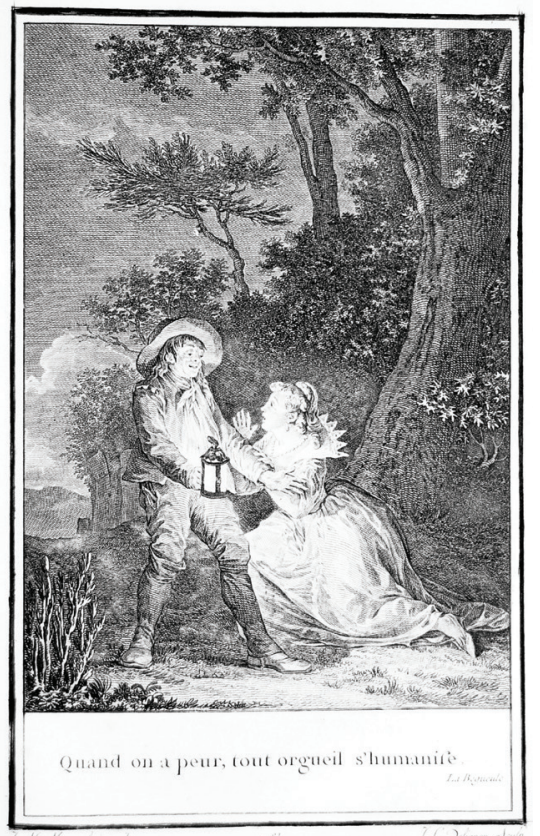

Fig. 3. - «Quand on a peur, tout orgueil s'humanise», La Bégueule, I783, Euvres complètes de Voltaire.

Société littéraire typographique, I784, tome I4 (collection privée).

Le merveilleux sert à éprouver l'héroïne, qui se lasse rapidement des nouveaux plaisirs. Une nouvelle intervention du merveilleux sert de punition : seule en plein bois, la bégueule rencontre un charbonnier qui lui donne l'hospitalité, en échange de ses faveurs. Moreau a illustré la rencontre, au moment où l'héroïne implore l'homme de la secourir. Un vers sert de légende, en forme de dicton : "Quand on a peur, tout

40. Voltaire, La Bégueule, ouvr. cité, p. 730. 
orgueil s'humanise ${ }^{41}$.» La forêt obscure compose le décor. La végétation est travaillée ici avec un art tout différent de la gravure précédente. À la végétation délicatement ciselée des jardins, composée d'essences rares et précieuses soigneusement choisies, s'oppose la forêt sauvage à la canopée tourmentée, aux troncs puissamment implantés dans le sol. Moreau exprime peut-être dans cette nature puissante le désir qui réclame ses droits, la sexualité inassouvie de la jeune femme à l'origine de toutes ses frustrations. Ce décor rend plus incongrue encore la parure de la jeune femme, placée au centre de la gravure. Moreau a joué sur les contrastes : la blancheur du costume d'Arsène ressortant sur la noirceur de l'arrière-plan. Quant au rustre, Moreau l'a campé en solide gaillard, un bras protecteur placé sur l'épaule de la jeune femme. Son expression concupiscente ne laisse pas de doute sur ses intentions. Heureusement, au moment crucial ou, ironie de Voltaire, "peut-être un peu $\operatorname{tard}^{42}$ », la fée ramène la belle Arsène en son logis. L'aventure sexuelle qu' elle a subie l'a ramenée à la raison. Assumant sa condition et ses désirs, elle prend un amant et devient «une femme accomplie ${ }^{43}$ ».

Ces trois premiers contes sont relativement proches par leur thématique. Voltaire explore les ressorts de la féminité. Avec Ce qui plaît aux dames, il revendique une participation des femmes au pouvoir et, avec Gertrude ou l'Éducation d'une fille et La Bégueule, une satisfaction naturelle du désir sexuel. Voltaire, dans ce domaine, fait preuve d'une grande modernité. Le merveilleux, puisant dans les modèles anciens de la fable ou du conte de fées, est porteur d'un discours et d'une réflexion sur la condition des femmes, même si l'épisode de la forêt reste ambigu.

Le Pauvre Diable est un conte moral moderne, lié à l'actualité littéraire des années 1760. Il fait partie des Contes de Guillaume Vadé. La fiction met en scène les tribulations d'un jeune homme qui cherche un métier, une condition, une place dans la société. Il appartient à ce que Robert Darnton nomme «la bohème littéraire ${ }^{44}$ ». Le conte prend la forme d'une conversation. Le "pauvre diable» s'interroge sur sa destinée, qui l'a conduit à tenter d'embrasser successivement les carrières de militaire, de magistrat, de religieux, de poète, sans succès :

Quel parti prendre? où suis-je et qui dois-je être?

Né dépourvu, dans la foule jeté,

4I. Ibid., p. 735 .

42. Ibid.

43. Ibid., p. 736.

44. R. Darnton, Bohème littéraire et révolution, Paris, Gallimard, I983. 
Germe naissant par le vent emporté,

Sur quel terrain puis-je espérer de craître?

Comment trouver un état, un emploi?

Sur mon destin, de grâce, instruisez-moi ${ }^{45}$.

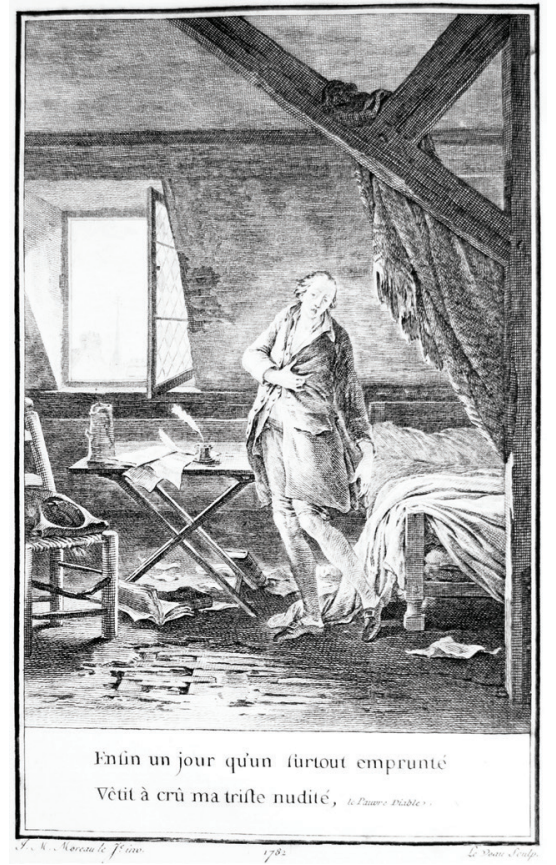

Fig. 4. - «Enfin un jour qu'un surtout emprunté vêtit à crû ma triste nudité... ", Le Pauvre Diable, 1782, Euvres complètes de Voltaire. Société littéraire typographique, I784, tome I4, [collection privée].

Dès l'incipit, Voltaire développe le thème du hasard de la destinée humaine, qui conduit un homme sans qualité à tenter la fortune au gré de rencontres douteuses. L'inégalité sociale est dénoncée par les mésaventures du héros. Seules la naissance et la richesse peuvent permettre d'accéder à une carrière honorable. Le passage du récit illustré par cette gravure correspond à celui où le jeune homme a tenté de devenir poète. Le «Parnasse »

45. Voltaire, Le Pauvre Diable, ouvr. cité, p. 320. Ce conte faisait partie du recueil des Contes de Guillaume Vadé, mais l'édition que nous utilisons a conservé la tradition éditoriale instituée par les éditeurs de Kehl, séparant ce morceau du recueil. La nouvelle édition des Euvres complètes de Voltaire, en cours de réalisation à la Voltaire Foundation d'Oxford, vient d'annoncer la prochaine parution de ce recueil, qui formera le tome $57 \mathrm{~B}$, publié sous la direction de S. Menant. 
se révèle vite "un enfer ${ }^{46}$ ". Le "pauvre diable» vit dans un «grenier» et couche "entre deux sales draps ${ }^{47}$ ». Moreau le Jeune a choisi d'illustrer la condition du misérable en le représentant dans une chambre sordide, sous les toits, aux murs et au sol dégradés par le manque d'entretien, suivant de près les indications du texte.

Le mobilier sommaire est lui aussi en mauvais état. Le désordre et la saleté ajoutent à ce tableau réaliste du dénuement. Sur la table, qui sert d'écritoire, quelques feuillets; au sol, des livres ouverts, «quelques méchants auteurs ${ }^{48}$ » qu'il a lus pour tenter d'y trouver l'inspiration, et de nombreux brouillons froissés et éparpillés témoignent du labeur du "pauvre diable». Sa silhouette se dresse au milieu de ce désordre. Le vers qui sert de légende, "Enfin un jour qu'un surtout emprunté/Vêtit à crû ma triste nudité ${ }^{49}$ ", éclaire la posture sous laquelle Moreau l'a représenté : il vient d'emprunter un vêtement pour se couvrir, et s'apprête à se rendre au café Procope pour y tromper sa faim et son ennui. Le visage du jeune homme, éclairé par la lumière que laisse passer la fenêtre ouverte sur les toits et les clochers de Paris, exprime son désespoir et son pessimisme. L'ensemble du tableau est particulièrement réaliste et constitue une exception dans la production de Moreau illustrateur de Voltaire, loin des décors de convention idéalisés - château médiéval, jardin ou forêt qui constituent le cadre des productions précédentes. La trivialité de la situation matérielle du personnage, de ses conditions de vie et de ses échecs successifs est rendue par la juxtaposition de la représentation du métier de poète et de la réalité de sa condition.

\section{Propositions de lectures}

Comment analyser ce corpus dans son ensemble? Quelle logique a guidé le choix de Moreau? Sur la trentaine de contes en vers contenus dans ce recueil, il a opéré une sélection et, à première vue, les quatre textes retenus pour l'illustration forment un ensemble hétérogène. Un regard plus attentif révèle cependant des pistes de lecture, révélatrices des choix effectués par Moreau.

46. Voltaire, Le Pauvre Diable, ouvr. cité, p. 323.

47. Ibid.

48. Ibid.

49. Ibid. 
L'intervention des fées est la principale manifestation du merveilleux dans Ce qui plaît aux dames et dans La Bégueule. Là encore, le parallèle a pu constituer un argument dans le choix des contes que Moreau a illustrés. Ces deux récits se fondent sur un schéma narratif identique : messire Robert et Dame Arsène se sont tous deux fourvoyés dans leurs premières amours. Tous deux errent à la recherche du bonheur. Les conventions sociales accordent aux hommes des amours passagères, uniquement destinées à satisfaire les exigences du corps, alors que pour les femmes, il faut accepter la loi du mariage et de la frustration sexuelle.

Le motif de l'initiation amoureuse a certainement constitué un premier point de départ : les deux premiers contes choisis par Moreau ont en effet pour thème l'initiation amoureuse d'un jeune homme et d'une jeune fille et peuvent constituer un premier diptyque. Dans Ce qui plaît aux dames, l'initiation de messire Robert, "un pauvre et noble chevalier ${ }^{50}$ ", se fait en deux temps : la découverte du plaisir charnel a lieu d'abord avec Marthon, qui le séduit à première vue par sa silhouette, puis avec la fée Urgèle, qu'il admire pour son esprit, son intelligence et avec qui il partage une complicité intellectuelle. D'une aventure à l'autre, le chevalier opère également une ascension sociale : après la brève liaison tarifée avec une paysanne, il épouse une fée qui lui apporte la richesse. Alors qu'il a dû rémunérer ses premiers plaisirs, il est récompensé par une dot. Dans le conte consacré à l'Éducation d'une fille, il n'est pas question de statut social. Seule la recherche du bonheur guide l'initiation amoureuse. Les plaisirs charnels sont essentiels à l'équilibre de la mère comme de la fille. La dimension morale de l'apologue est plus importante ici, renversant l'ordre des valeurs et faisant de la satisfaction des désirs féminins une condition essentielle du bonheur et un impératif social, n'en déplaise aux injonctions de la morale chrétienne. Le dénouement est plus conventionnel dans le premier conte que dans le second, mais ce qui a peut-être intéressé Moreau ici, c'est le thème de la jeunesse et de l'innocence. Voltaire décline l'initiation amoureuse en deux modalités distinctes : le hasard d'une découverte, l'instinct et l'intelligence conduisent très rapidement la jeune fille à son autonomie et à son émancipation. Pour le jeune garçon, les choses sont plus complexes, il se laisse piéger par les mirages de l'apparence, il est naif et son initiation amoureuse est semée d'embûches. L'intervention du merveilleux est nécessaire pour le conduire sur la voie du bonheur.

50. Voltaire, Ce qui plaît aux dames, ouvr. cité, p. 367. 
Le sujet de l'illustration choisi par Moreau, dans les deux cas, est le moment du récit où le protagoniste se trouve au seuil de son initiation. Avec l'arrivée de la vieille, le jeune homme, qui croyait conclure son aventure par la fin de son procès, est projeté dans une autre dimension, celle du merveilleux qui le conduira à la découverte du véritable amour, celui qui associe idéalement beauté des corps, harmonie des esprits et des conditions sociales. Dans l'Éducation d'une fille, le dessin figure la révélation du secret de la mère, qui dissimule sa liaison amoureuse avec un inconnu. En écoutant à la porte les soupirs de plaisir et les paroles de reconnaissance, la jeune Isabelle accède elle aussi au merveilleux, à la connaissance du secret qui conduit au bonheur, au moment précis où ses sens commençaient à s'éveiller.

En illustrant successivement l'Éducation d'une fille et La Bégueule, les deux contes qui mettent en scène l'initiation de la femme à l'Éros, Moreau suggère là encore une lecture en diptyque, soulignant la complémentarité des deux fables. L'éducation d'Isabelle n'est entravée par aucun obstacle, ce qui permet à l'héroïne d'accéder rapidement à une forme de bonheur fondée sur une liberté sexuelle. Dans le cas d'Arsène, l'initiation se fait après un premier échec. L'intervention du merveilleux a pour but de la mettre à l'épreuve. La révélation se fait sur le mode de la violence, hors du monde des conventions sociales. Le passage par l'affreux désert et la forêt sauvage est nécessaire pour retrouver la clé du bonheur. Le rustre lui fait découvrir, malgré elle, sa véritable nature.

L'initiation de héros masculins à la vie sociale constitue, symétriquement, le thème des deux autres contes, Ce qui plaît aux dames et Le Pauvre Diable. À travers les aventures amoureuses, judiciaires et philosophiques de messire Robert, découvrant successivement la malice des hommes et des femmes, les règles sociales, les conventions de la cour, tour à tour victime et accusé, soumis à une série d'épreuves destinées à lui faire acquérir un savoir et un jugement, à soumettre sa raison à l'épreuve des faits et de l'expérience, toute une éducation se décline symboliquement dans les aventures guidées par deux femmes puissantes, une reine et une fée, qui président sagement à sa destinée et le soumettent à une initiation sociale et sentimentale. Dans Le Pauvre Diable, l'initiation sociale repose sur des données pragmatiques. Face à un système de privilèges et de castes, les aspirations professionnelles et personnelles du jeune homme sont freinées par la trivialité de la réalité. Le parcours du héros est une déchéance sociale qui le conduit au désespoir. Aucune fée pour le guider ni pour favoriser sa quête dans ce récit réaliste de la misère des conditions humaines. Seul un enchaînement de circonstances dues au hasard des rencontres lui permet 
de persévérer dans sa tentative de trouver un état. Si Voltaire a profité de ce récit pour régler ses comptes avec la canaille des journalistes et des littérateurs sans foi ni loi, il a surtout illustré de façon particulièrement crue une réalité sociale et économique, faisant du Pauvre Diable une satire de l'opportunisme et de l'amoralité engendrés par un système social inégalitaire.

C'est tout le fonctionnement de la poétique voltairienne qui se donne à lire dans ces contes : l'art doit être à la fois agréable et utile. Les contes merveilleux possèdent, eux aussi, une valeur philosophique. "Quand j'ai du chagrin, je m'amuse à faire des contes", aimait à confesser Voltaire ${ }^{\varsigma 1}$. Mais la fiction n'a pas pour seule fonction d'amuser le lecteur. Dans un poème intitulé "Apologie de la fable», l'auteur se réclame d'Hésiode car "Par lui l'esprit humain s'éclaire ${ }^{52}$ ». Il rend hommage à l'héritage antique porteur de vérités :

Savante antiquité, beauté toujours nouvelle,

Monument du génie, heureuses fictions,

Environnez-moi des rayons

De votre lumière immortelle ${ }^{53}$.

Dans un premier essai intitulé Sur la fable, datant de 1745 , Voltaire avait défini sa relation aux fables et à la fiction. Il s’inscrit dans une démarche philosophique épicurienne :

Lucrèce ne croyait pas à ces dieux de la fable; mais il célébrait la nature sous le nom de Vénus. [...] Les belles fables de l'antiquité ont encore ce grand avantage sur l'histoire, qu'elles présentent une morale sensible : ce sont des leçons de vertu; et presque toute l'histoire est le succès des crimes ${ }^{54}$.

Toute la démarche des contes merveilleux est contenue dans cette interrogation :

Une fiction qui annonce des vérités intéressantes et neuves n'est-elle pas une belle chose? [...] Pour les fictions qui ne figurent rien, qui n'enseignent rien, dont il ne résulte rien, sont-elles autre chose que des mensonges? et si elles sont incohérentes, entassées sans choix, comme il y en a tant, sont-elles autre chose que des rêves ${ }^{5}$ ?

5I. Voltaire à Chabanon, II mai 1772, D 17736.

52. Voltaire, «Apologie de la fable», article "Fable», Questions sur l'Encyclopédie, t. V, CEuvres complètes, t. 4I, C. Mervaud et N. Cronk (éd.), Oxford, Voltaire Foundation, 20IO, p. 324.

53. Ibid., p. 322-323.

54. Voltaire, Sur la fable, D. Williams (éd.), Euvres complètes de Voltaire, t. 28B Writings of I742I745 (II), p. 216-218.

55. Voltaire, Questions sur l'Encyclopédie, article «Fiction», ouvr. cité, p. 390-39I. 
Voltaire partait d'un constat : "Les hommes aimant naturellement les images et les contes ${ }^{56}$ ", "ces images vraies et riantes ${ }^{57}$ ». Il s'agit donc de «mentir habilement ${ }^{58}$ » et surtout utilement. Face aux «fanatiques qui ont voulu proscrire les anciennes fables ${ }^{59}$ ", il rétorque : "Il serait triste pourtant de brûler Ovide, Homère, Hésiode, et toutes nos belles tapisseries, et nos tableaux, et nos opéras : beaucoup de fables, après tout, sont plus philosophiques que ces messieurs ne sont philosophes ${ }^{60}$." Les arguments esthétiques le disputent aux arguments éthiques :

Je sais combien l'histoire peut nous instruire, je sais combien elle est nécessaire; mais en vérité il faut lui aider beaucoup pour en tirer des règles de conduite. [...] Pour qui ne regarde qu'aux événements, l'histoire semble accuser la providence, et les belles fables morales la justifient. Il est clair qu'on trouve dans elles l'utile et l'agréable : ceux qui dans ce monde ne sont ni l'un ni l'autre crient contre elles ${ }^{61}$.

Ces quelques éléments aident à saisir l'enjeu esthétique et philosophique de la poétique des contes voltairiens. Par ses choix littéraires et son travail d'interprétation graphique, Moreau illustre la fiction par des propositions de lecture qui soulignent, prolongent et amplifient les valeurs suggérées par le récit. Les censeurs n'ont pas manqué de relever le danger des propositions axiologiques contenues dans les contes voltairiens. Â l'heure où les éditeurs s'attachaient à rassembler l'intégralité du corpus voltairien, construisant ainsi un patrimoine littéraire et philosophique fondateur de la réception de l'œuvre de Voltaire, une série de mandements épiscopaux, complétés par une censure théologique de la Sorbonne et un Arrêt royal ordonnant la suppression de l'édition se déchaînent contre l'entreprise ${ }^{62}$. Dès 178I, l'évêque d'Amiens fustigeait ainsi l'édition annoncée, mettant particulièrement l'accent sur le danger de la lecture des contes :

Personne n'ignore que parmi les mauvais livres dont la France est inondée, malgré les plaintes \& les gémissements de tous les gens vertueux, beaucoup d'ouvrages du Sir de Voltaire tiennent le premier rang \& ont opéré une affreuse corruption[...]. En effet personne n'a plus malignement saisi que cet abominable Auteur, l'art de séduire dans un siècle frivole et libertin comme celui ci. Pour attaquer, comme il a

56. Voltaire, Questions sur l'Encyclopédie, article «Fable», ouvr. cité, p. 313.

57. Voltaire, Sur la fable, ouvr. cité, p. 216.

58. Voltaire, Questions sur l'Encyclopédie, article «Fable», ouvr. cité, p. 315.

59. Ibid., p. 32I.

6o. Voltaire, Sur la fable, ouvr. cité, p. 215.

6I. Ibid., p. 218-219.

62. Voir à ce sujet notre contribution : "Le "Grand Voltaire de Kehl” à la conquête de sa légitimité", Krypton, $\mathrm{n}^{\circ}$ 2, Rome, Presses de l'université de Roma Tre, 2013,

$<$ http://host.uniroma3.it/progetti/romatrepress/Krypton/Krypton_II/Krypton_II_o6.pdf>. 
fait, tous les principes de la religion et des mœurs, ce ne sont pas les raisonnements qu'il a employés; outre qu'on en sentirait la faiblesse, il savait que le grand nombre des lecteurs n'aime point l'étude ni les discussions sérieuses. Il a donc accumulé les dérisions, les contes, les épigrammes; les a ornés d'une diction agréable et brillante; il a subjugué par là des esprits superficiels, des gens voluptueux et passionnés qui trouvent plus aisé de mépriser une religion qui les gêne, que d'examiner attentivement sur quoi sont fondés ses préceptes et ses menaces. La prétention qu'avait le Sieur de Voltaire aux honneurs de génie universel lui a fait exercer sa plume dans presque tous les genres, et il a porté partout l'empreinte de ses ravages ${ }^{63}$.

Nul doute dans ce contexte que les figures de Moreau le Jeune n'aient constitué, pour les censeurs, un argument supplémentaire dans l'opposition à la diffusion de cette œuvre subversive. L'œuvre gravée, augmentant la portée de l'œuvre textuelle, témoigne d'une conscience aiguë des enjeux complexes de cette édition et de la réception posthume des contes de Voltaire.

63. L.-C. de Machault, Mandement de Monseigneur l'Évêque d'Amiens, Au sujet de l'annonce publiée dans son Diocèse d'une Collection entière des ouvres du Sieur de Voltaire, Amiens, 9 avril I78I, dans Les Voltairiens, J. Vercruysse (éd.), Nendeln, KTO Press, 1978, 8 vol., t. III, nº I2. 